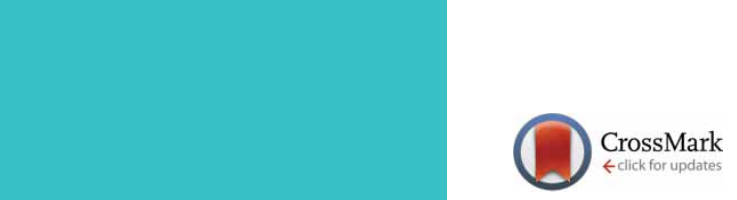

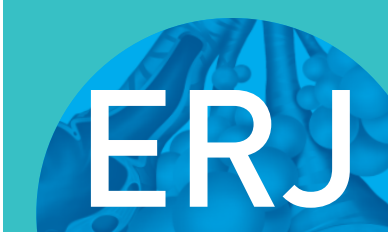

open research

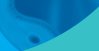

\title{
Does prior mediastinal lymph node aspiration contribute to false-positive positron emission tomography-computed tomography?
}

\author{
J. Michael Ramsahai ${ }^{1}$, Christine Molnar ${ }^{2}$, Lawrence Lou ${ }^{2}$, Winston Ying ${ }^{2}$, \\ Paul MacEachern ${ }^{1}$, Christopher A. Hergott ${ }^{1}$, Elaine Dumoulin ${ }^{1}$, \\ Nadine Strilchuk ${ }^{1}$, Marc Fortin $^{1}$ and Alain Tremblay ${ }^{1}$
}

Affiliations: ${ }^{1}$ Division of Respiratory Medicine, Cumming School of Medicine, University of Calgary, Calgary, AB, Canada. ${ }^{2}$ Dept of Medical Imaging, Alberta Health Services, Calgary, AB, Canada.

Correspondence: Alain Tremblay, 3300 Hospital Drive NW, Calgary, AB, T2N4N1, Canada. E-mail: atremblaA ucalgary.ca

\section{ABSTRACT}

Background: Proper staging of the mediastinum is an essential component of lung cancer evaluation. Positron emission tomography-computed tomography (PETCT) and endobronchial ultrasound transbronchial needle aspiration (EBUS-TBNA) are an integral part of this process. False-positive PETCT results can occur following surgical procedures but has not been demonstrated following EBUS-TBNA. We aimed to determine whether false-positive PETCT rates increase when EBUS-TBNA is performed prior to PETCT.

Study design and methods: A retrospective review was carried out of clinical cases that underwent both PETCT and EBUS-TBNA within 30 days for the suspected malignancy. The impact of test sequence on the PETCT false-positive rate (FPR) was determined using Generalised Estimating Equation logistic regression analysis.

Results: A total of 675 lymph node stations were sampled and imaged on PETCT. Overall, 332 (49.2\%) nodes were sampled by EBUS-TBNA before PETCT, and 343 (50.8\%) afterwards, with the interval between EBUS and subsequent PETCT being a mean \pm SD of $11.6 \pm 6.8$ days (range 1-29). The FPR on qualitative PETCT for the EBUS first group was 41 (23.2\%) out of 164, and for PETCT first it was 57 (29.0\%) out of 193 for a difference of $5.8 \%$ (95\% CI $-3.4-14.7, \mathrm{p}=0.22)$. In the regression model, EBUS as the first test was associated with a lower FPR when using the clinical PETCT interpretation.

Interpretation: The performance of EBUS-TBNA sampling did not influence the FPR of PETCT when bronchoscopy took place in the 30 days prior to testing. Test sequence should be selected based on other clinical considerations.

@ERSpublications

In patients with suspected malignancy undergoing endobronchial ultrasound transbronchial needle aspiration (TBNA) as well as PETCT scan, performing the TBNA prior to PETCT does not increase the rate of false-positive PET scan results https://bit.ly/3gFy6Fi

Cite this article as: Ramsahai JM, Molnar C, Lou L, et al. Does prior mediastinal lymph node aspiration contribute to false-positive positron emission tomography-computed tomography? ERJ Open Res 2020; 6: 00103-2020 [https://doi.org/10.1183/23120541.00103-2020].

This article has supplementary material available from openres.ersjournals.com

Received: 28 Feb 2020 | Accepted: 3 July 2020

Copyright $\odot$ ERS 2020. This article is open access and distributed under the terms of the Creative Commons Attribution Non-Commercial Licence 4.0. 


\section{Introduction}

Lung cancer remains the leading cause of cancer deaths among both men and women. The majority of cases are due to nonsmall cell lung cancer [1]. Proper staging is essential to ensure the right therapy for each individual with staging of the mediastinum comprising a key decision point.

Positron emission tomography-computed tomography (PETCT) scanning is now an integral part of the diagnosis and staging of lung cancer, alongside invasive staging of the mediastinum [2]. Given the implications of treatment decisions based on this information, invasive staging remains necessary due to the relatively lower accuracy of PETCT staging [3]. To conduct invasive staging, sampling of lymph nodes is now routinely performed with endobronchial ultrasound transbronchial needle aspiration (EBUS-TBNA) [4]. While there has been clear documentation of false-positive results in PETCT scanning in the setting of surgical procedures, this has not been demonstrated with respect to EBUS-TBNA [5, 6]. Uncertainty surrounding this question means that patients may face unnecessary delay while clinicians try to secure a particular sequence of testing when investigating possible chest malignancy. In order to provide further clarity on the appropriate sequence of testing, we set out to determine whether EBUS-TBNA of hilar and mediastinal lymph nodes would result in false-positive findings on subsequent PETCT. We hypothesised that an increased false-positive rate (FPR) of nodal stations in PETCT would occur if EBUS-TBNA is performed first, due to biopsy-induced inflammation or trauma.

\section{Materials and methods}

We conducted a retrospective review using the Alberta Thoracic Oncology Program (ATOP) database of patients that were referred for suspicion of intrathoracic malignancy in our centre. This consisted of records from all patients $(n=703)$ who had undergone assessment for possible chest malignancy by the ATOP physicians from December 1, 2010 to August 31, 2015 at the Foothills Medical Centre in Calgary, Canada. This is a tertiary referral centre in Western Canada with a catchment area of approximately two million people, living in Southern Alberta, Eastern British Columbia and Western Saskatchewan. The study was approved by the Health Research Ethics Board of Alberta Cancer Committee (CC-16-0811).

Records of patients undergoing EBUS-TBNA and PETCT examination within 30 days of each other and a pre-procedural suspicion of lung malignancy were selected for analysis. Information pertaining to patient demographics, testing date, tumour size, stage, lymph node station and qualitative fluorodeoxyglucose (FDG) avidity (standardised uptake values (SUVs) are not routinely recorded in our institution; scans are reported as: negative; low uptake (less than mediastinum); moderate uptake (greater than mediastinum but less than liver); high uptake (equal or greater than liver)) on PETCT, EBUS-TBNA pathological diagnosis, and any evidence of subsequent confirmatory testing (surgical biopsy, CT evidence of growth within the next 3 months as per clinical radiology reports or higher stage lymph nodes with positive malignant pathology) was extracted from the clinical records.

EBUS procedures were performed under moderate sedation with linear ultrasound endoscopes (BF-UC160f or 180f; Olympus Canada, Markham, ON, Canada) and a dedicated 21G EBUS-TBNA needle (NA-201SX-4021; Olympus Canada). Typically, when EBUS is performed in a "staging" setting, all accessible lymph nodes $\geqslant 5 \mathrm{~mm}$ are targetted. If EBUS is used for primarily diagnostic purposes in patients with clear evidence of advanced disease, the most suspicious nodes will be targetted at operator discretion. Rapid on-site evaluation of specimens was not performed. Pathology interpretation was classified as malignant, benign (presence of lymphocytes/representative of a lymph node) or inadequate/ non-diagnostic.

All PETCT examinations were reviewed by one of four study nuclear medicine radiologists without knowledge of cytopathological results or final stage. Each lymph node of interest was measured (short axis), FDG avidity determined qualitatively as per local clinical practice (negative; low; moderate; high) and SUV measurements taken (volume of interest (VOI), maximum). SUV was also recorded for the primary lesion (VOI, maximum), mediastinal blood pool and liver (VOI, mean). Scans were performed on one of two machines: Biograph 16 and Biograph MCT (Siemens Health Care Ltd, Oakville, ON, Canada). A random sample of $10 \%$ of cases were interpreted once again by one of the study radiologists.

All sampled lymph node stations were assessed to determine if PETCT interpretation represented a false-positive) result or true-negative result on a per-node basis. For the primary analysis, a false positive was defined as a lymph node with moderate or high intensity FDG uptake on initial clinical PETCT interpretation, but negative for malignancy on EBUS-TBNA sampling and without evidence of subsequent positive biopsy (repeat EBUS-TBNA or surgical) or growth on subsequent CT imaging, or evidence of higher stage nodal involvement with malignancy. Secondary analyses were also performed with four alternative definitions of PETCT positivity: qualitative research review; quantitative lymph node SUV>mediastinal blood pool uptake; quantitative lymph node SUV>liver uptake; and qualitative review 
but not considering cases where "symmetric bilateral nodal uptake consistent with inflammatory process" was noted as false-positive. True-negative lymph nodes were those with negative or low FDG intensity on clinical interpretation (as well as the opposite of the alternative secondary definitions) and also meeting the criteria for the absence of malignant involvement described above. The FPR was then calculated as false-positive/(false-positive+true-negative).

Continuous variables were described using the mean and standard deviation (SD) for approximately normally distributed variables and the median and interquartile range for skewed variables. The distributions of categorical variables were described using $\mathrm{n} / \mathrm{N}$ and percentages. Interobserver agreement in qualitative PET assessment dichotomised as negative (negative/low uptake) or positive (moderate or high uptake) was performed between the clinical interpretation and the research review, and between the two research reviews in the randomly selected $10 \%$ of cases. Raw FPRs were calculated as a percentage and the difference between the EBUS first and PETCT first group calculated along with the 95\% confidence interval (95\% CI) around this difference. To account for intra-subject correlation as more than one lymph node per patient could be present, and to adjust for other factors that may affect FPR, binary Generalised Estimating Equation (GEE) logistic regression models of FPRs were generated as follows. Several potential predictor variables (PPVs) at both the patient and the lymph node level were also considered. At the patient level the variables were age, sex, time interval between the EBUS and PETCT examinations, and presence of a bilateral symmetric inflammatory lymph node pattern on PETCT. At the lymph node level the variables were lymph node station (re-grouped into three zones: stations 2 \& 4: paratracheal; station 7: subcarinal; stations $\geqslant 10$ : hilar/pulmonary), lymph node size and lymph node SUVs. For each PPV, an initial model was generated including only that predictor variable to assess whether there was an effect of that variable on FPR. Subsequently an interaction term between the PPV of interest and test sequence (EBUS first versus PETCT first) was added to the model including both test sequence and the PPV as main effects. PPVs and interactions with significant Wald Chi-squared values at the $\mathrm{p} \leqslant 0.1$ level were included in the final model. FPRs were also calculated for each PPV according to the sequence of testing EBUS-TBNA first versus PETCT first, using the median as a cut-off for continuous PPVs.

Sample size was determined based on a desire to notice a $20 \%$ clinically significant difference in the FPR of PETCT, assuming a priori a rate of $20 \%$ [3]. Assuming that the majority of patients would have 1-4 nodes sampled per procedure, and with analysis to be done on a per nodal basis, this yielded a necessity for 109 nodes in each test sequence group (total 218) to provide $90 \%$ power at the $5 \%$ level of significance to detect a difference between $20 \%$ and $40 \%$. In order to account for intra-patient dependence between nodes sampled from the same patient, this was multiplied by a Design Effect coefficient to yield a total of 406 nodes needed.

\section{Results}

The records of 703 patients in the ATOP database were screened for inclusion, with 214 patients identified who had had both EBUS-TBNA and PETCT performed within 30 days of each other, yielding a total of 731 lymph nodes. We analysed a total of 675 lymph node stations sampled in those patients who had a final diagnosis of cancer. Of these, 332 (49.2\%) nodes were sampled by EBUS-TBNA before their PETCT, and $343(50.8 \%)$ afterwards. Time between EBUS and subsequent PETCT was a mean \pm SD of $11.6 \pm 6.8$ days (range 1-29). Fourteen out of 675 (2\%) EBUS node stations sampled were interpreted as inadequate. These were small nodes (mean $4.8 \mathrm{~mm}$ ), and none of these nodes was noted to be of moderate or high PET uptake and as such did not influence the PET false-positive rate. Agreement in PETCT interpretation was substantial (kappa 0.67 ) between the qualitative clinical interpretations and research review, and moderate (kappa 0.50 ) between the two research reviews for the randomly selected $10 \%$ subset of examinations.

Table 1 presents the demographic data for the patients selected, with no significant differences detected between the groups. Given that the calculation of FPR is only dependent on false-positive and true-negative nodes (i.e. disease-negative nodes), true-positive and false-negative nodes (i.e. disease-positive nodes) are not considered further in this analysis. Table 2 outlines characteristics of 399 individual disease-negative lymph nodes included in the analysis. Lymph nodes in the EBUS first group were slightly larger than in the PETCT group $(7.5$ versus $6.7 \mathrm{~mm}$ ) and were associated with higher mean SUV of the primary pulmonary lesion. Characteristics of the false-positive nodes are outlined in table 3, with only primary lesion SUVs being different between the two groups. All EBUS specimens in the false-positive group were adequate, showing lymphocytes in 97 out of 98 (99\%) cases and granuloma in one lymph node. Few lymph nodes labelled as false-positive underwent additional cytopathological confirmation via repeat EBUS, mediastinoscopy or surgical sampling at time of resection, but this was similar in each group (6 out of $41(14.6 \%)$ in EBUS first group and 8 out of 57 (14.0\%) in PET first group).

The overall raw FPR on clinical qualitative PETCT was 98 out of 399 (24.6\%, 95\% CI 20.4-29.1). The FPR for EBUS first group was 41 out of $184(23.2 \%)$ whereas for the PETCT first group it was 57 out of 


\section{TABLE 1 Demographics of study participants in each study group}

EBUS first

Subjects $\mathrm{n}$
Age years
Male sex
Ever smoker
Lymph node stations sampled $\mathrm{n}$
Interval between tests days
Final diagnosis
Nonsmall cell lung cancer
Small cell lung cancer
Other malignancy
Cancer stage
I
II
III
IV

$\begin{array}{cc}107 & 107 \\ 70.0 \pm 10.1 & 70.0 \pm 10.6 \\ 59(55.1) & 49(45.8) \\ 94(87.9) & 92(86.0) \\ 3 \pm 1.2 & 3 \pm 1.4 \\ 11.6 \pm 6.8 & 11.0 \pm 8.7 \\ & \\ 93(86.9) & 90(84.1) \\ 7(6.5) & 8(7.5 \%) \\ 7(6.5) & 9(8.4 \%) \\ 17(15.9) & \\ 14(13.1) & 33(30.8) \\ 44(41.1) & 18(16.8) \\ 32(29.9) & 31(29.0) \\ & 25(23.4)\end{array}$

Data are presented as mean \pm SD or $\mathrm{n}(\%)$, unless otherwise stated. EBUS: endobronchial ultrasound; PETCT: positron emission tomography-computed tomography.

$215(26.5 \%)$ for a difference of $4.2 \%(95 \%$ CI $-4.3-12.5$, p=0.33). No significant differences in FPR between groups were noted with three of the secondary false-positive definitions, but for the estimates which excluded cases of bilateral inflammatory pattern on PETCT as false-positive, the EBUS first group had a higher FPR of 43 out of 184 (23.4\%) versus 33 out of 215 (15.3\%); difference $-8.1 \%$ (95\% CI $0.36-15.9, \mathrm{p}=0.04)$ (table 4).

FPRs associated with each potential predictive variable and results of the initial GEE modelling for the effect of each variable and the interaction of those variables with the testing sequence are presented in supplementary Table S1. In the final GEE model, undergoing EBUS as the first test was associated with a lower rate of false-positive results. Regression coefficients and statistical significance for each variable in the model are presented in table 5. Secondary analysis repeating the GEE regression modelling using the qualitative PETCT review results and the lymph node SUV>mediastinum SUV or lymph node SUV $>$ liver SUV definitions as well as excluding cases of bilateral inflammatory pattern on PETCT as false-positive in

\section{TABLE 2 Disease negative (benign) lymph node characteristics in each study group}

\begin{tabular}{|c|c|c|}
\hline & EBUS first & PETCT first \\
\hline Subjects $n$ & 184 & 215 \\
\hline Size $\mathrm{mm}^{*}$ & $7.5 \pm 3.5$ & $6.7 \pm 2.7$ \\
\hline \multicolumn{3}{|l|}{ Nodal station } \\
\hline Paratracheal & 69 (37.7) & $70(36.2)$ \\
\hline Subcarinal & $46(25.1)$ & $46(21.4)$ \\
\hline Hilar/pulmonary & 68 (37.2) & $99(46.0)$ \\
\hline \multicolumn{3}{|l|}{ Quantitative SUV } \\
\hline Lymph node & $0.9 \pm 1.1$ & $1.0 \pm 1.1$ \\
\hline Primary lesions* & $14.4 \pm 7.0$ & $11.8 \pm 8.2$ \\
\hline \multicolumn{3}{|c|}{ Qualitative PETCT uptake on clinical interpretation } \\
\hline Negative & $91(49.5)$ & 93 (43.3) \\
\hline Low & 52 (28.3) & $65(30.2)$ \\
\hline Moderate & $31(16.8)$ & $39(18.1)$ \\
\hline High & $10(5.4)$ & $18(8.4)$ \\
\hline \multicolumn{3}{|l|}{ EBUS-TBNA results } \\
\hline Lymphocytes & 168 (91.3) & 203 (94.4) \\
\hline Granuloma & $3(1.6)$ & $4(1.9)$ \\
\hline Inconclusive/inadequate & $13(7.1)$ & 8 (3.8) \\
\hline \multicolumn{3}{|c|}{$\begin{array}{l}\text { Data are presented as mean } \pm \text { SD or } n(\%) \text {, unless otherwise stated. EBUS: endobronchial ultrasound; } \\
\text { PETCT: positron emission tomography-computed tomography; SUV: standardised uptake value; } \\
\text { EBUS-TBNA: endobronchial ultrasound-transbronchial needle aspiration. }{ }^{*}: p<0.05 \text {. }\end{array}$} \\
\hline
\end{tabular}




\begin{tabular}{|c|c|c|}
\hline & EBUS first & PETCT first \\
\hline Subjects $\mathrm{n}$ & 41 & 57 \\
\hline Size mm & $8.6(4.5)$ & 7.4 (2.9) \\
\hline \multicolumn{3}{|l|}{ Nodal station } \\
\hline Paratracheal & $10(24.4)$ & $21(36.8)$ \\
\hline Subcarinal & $8(19.5)$ & $9(15.8)$ \\
\hline Hilar/pulmonary & $23(56.1)$ & $27(47.4)$ \\
\hline \multicolumn{3}{|l|}{ Quantitative SUV } \\
\hline Lymph node & $4.43 \pm 1.87$ & $4.36 \pm 1.72$ \\
\hline Primary lesions* & $13.29 \pm 7.68$ & $8.46 \pm 6.51$ \\
\hline \multicolumn{3}{|c|}{ Qualitative PETCT uptake on clinical interpretation } \\
\hline Moderate & $31(75.6)$ & 39 (68.4) \\
\hline High & $10(24.4)$ & $18(31.6)$ \\
\hline \multicolumn{3}{|l|}{ EBUS-TBNA results } \\
\hline Lymphocytes & $40(97.5)$ & 57 (100) \\
\hline Granuloma & $1(2.4)$ & \\
\hline \multicolumn{3}{|c|}{ Inconclusive/inadequate } \\
\hline \multicolumn{3}{|c|}{$\begin{array}{l}\text { Data are presented as } n(\%) \text { or mean } \pm \mathrm{SD} \text {, unless otherwise stated. EBUS: endobronchial ultrasound; } \\
\text { PETCT: positron emission tomography-computed tomography; SUV: standardised uptake value; } \\
\text { EBUS-TBNA: endobronchial ultrasound-transbronchial needle aspiration. } *: p<0.05 \text {. }\end{array}$} \\
\hline
\end{tabular}

our definition of PETCT positivity did not demonstrate a significant impact of test sequence on FPR (data not shown).

\section{Discussion}

This retrospective study examined the FPR of PETCT in patients undergoing EBUS-TBNA prior to or after this test. We found no significant evidence that EBUS-TBNA prior to PETCT is associated with an increased PETCT false-positive rate based on initial PETCT clinical interpretation. This finding was consistent when using three alternative definitions for PETCT positivity. Raw FPRs were higher in the EBUS first group when applying a fourth definition which excluded a bilateral inflammatory pattern as false-positive. Potentially the bronchoscopists were more inclined to sample such nodes after PETCT which otherwise would not have been targetted and as such increased the FPR in the PETCT first group. We also noted a trend to higher FPR in early stage lung cancer patients who underwent EBUS first. On the other hand, the presence of a bilateral inflammatory pattern was not a significant factor affecting FPR in the multivariate GEE model. These findings suggest that overall, EBUS-TBNA performed within 30 days of PETCT is not a significant contributor to false-positive PETCT.

False-positive PETCT findings have been described in association with biopsy and surgical sites, prostheses, areas of drainage, port or catheter insertion, and along injection sites (particularly those associated with insulin or colony-stimulating factor) [5,6]. Given that false-positive SUV uptake can be seen in association with small insults, such as areas of insulin injection or biopsy sites, it is not unwarranted to consider whether this may be the case following EBUS-TBNA. The consequences of a false-positive PETCT result are not insignificant and include the possibility of incorrect stage determination, need for additional, more invasive, testing, and potentially incorrect treatment decisions due to incorrect upstaging.

TABLE 4 Raw false-positive rates according to PETCT positivity criteria

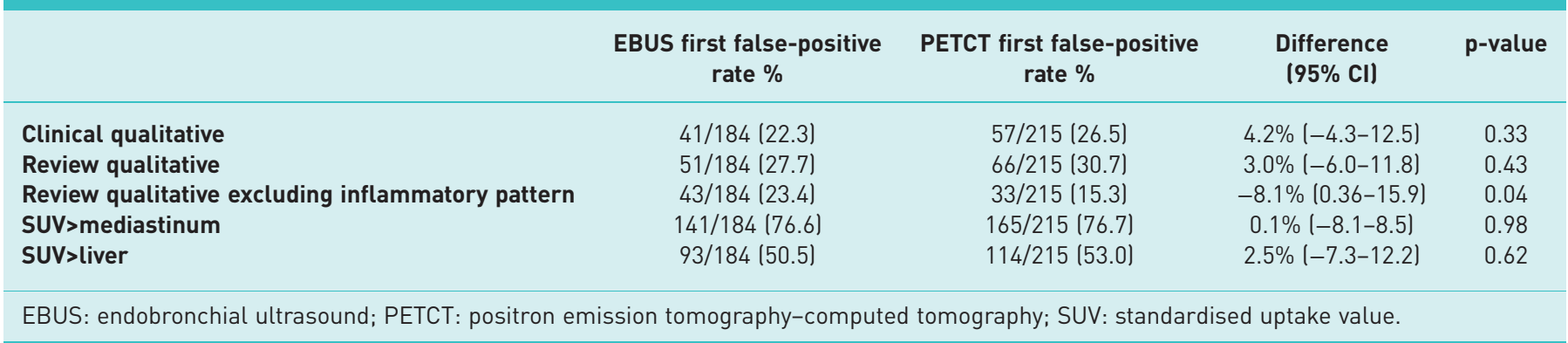




\begin{tabular}{|c|c|c|c|c|}
\hline \multirow[t]{2}{*}{ Parameter } & \multirow[t]{2}{*}{ B } & \multicolumn{3}{|c|}{$95 \%$ Wald $\mathrm{Cl}$} \\
\hline & & Lower & Upper & Sig. \\
\hline (Intercept) & 6.584 & 3.167 & 10.000 & 0.000 \\
\hline EBUS first & -4.613 & -6.868 & -2.358 & 0.000 \\
\hline Age years & -0.014 & -0.050 & 0.022 & 0.450 \\
\hline Days between tests & -0.015 & -0.056 & 0.026 & 0.478 \\
\hline Lymph node size mm & -0.061 & -0.183 & 0.061 & 0.329 \\
\hline Node SUV & -1.398 & -2.005 & -0.791 & 0.000 \\
\hline Paratracheal stations & -0.671 & -1.780 & 0.438 & 0.235 \\
\hline Subcarinal station & 0.082 & -1.196 & 1.360 & 0.900 \\
\hline Absence of symmetric uptake & 0.817 & -0.124 & 1.757 & 0.089 \\
\hline \multicolumn{5}{|l|}{ Interaction terms } \\
\hline EBUS first * paratracheal stations & 2.296 & 0.892 & 3.701 & 0.001 \\
\hline EBUS first * subcarinal station & 1.291 & -0.229 & 2.810 & 0.096 \\
\hline EBUS first * node SUV & 1.146 & 0.481 & 1.810 & 0.001 \\
\hline
\end{tabular}

Lung cancer staging guidelines do not specify if an ideal sequence exists with regard to PETCT and invasive mediastinal staging nor if there exists a need for any delay between biopsy and PETCT. In the American College of Chest Physicians (ACCP) guidelines, both CT and PETCT results are used to determine which patients should undergo invasive mediastinal staging, such as EBUS [2]. When this is indicated based on CT results, however, it is unlikely PETCT will preclude more invasive testing unless occult metastatic disease is detected. Should PETCT confirm metastatic disease, the bronchoscopic procedure may then have already provided the important cytopathological and molecular information required for treatment decisions. As such, proceeding to minimally invasive mediastinal diagnosis and staging with EBUS seems a reasonable path for some patients, particularly if PETCT availability is delayed by health system factors such as is common in our setting. Confirming that this sequence does not hinder the test performance characteristics of the PETCT would remove one of the concerns and causes for delay with this approach.

Only limited published evidence exists on this topic. A small study of 27 patients who underwent CT-guided lung biopsy failed to show any abnormal PETCT abnormalities in the chest wall/needle track area traversed on scans performed within 14 days [7] suggesting that the small injury from a needle sample may not be enough to impact PETCT results. The only study examining PETCT results following EBUS procedures in a cohort of 107 patients with 198 non-malignant lymph nodes found that subsequent PETCT was positive by visual interpretation in 76 lymph nodes (38\%) [8]. The authors felt that no other explanation for the PETCT positivity other than the biopsy was present in $14 \%$ of cases. Another study published in abstract form only found a false-positive PETCT prevalence of $31 \%$ in 71 patients when imaging was performed after EBUS sampling [9]. But since these studies have no control groups, PETCT is known to have a significant FPR [2] and explanations for this are not always evident, attributing any false-positive results to the EBUS procedure would be tenuous at best.

Our study reports on the largest collection of PETCT cases preceded by EBUS but also allowed us to compare this to a group where EBUS-TBNA was performed after PETCT to more objectively determine any impact of the EBUS procedure on FPRs. Using several approaches to classify PETCT positivity including the initial clinical qualitative reports, qualitative and quantitative research re-interpretation of scans, and using a standardised definition of malignant lymph node involvement, we found no evidence that performing EBUS prior to PETCT influenced the false-positive rate. It should be noted that our estimates of PETCT FPR may not be accurate as stand-alone values, but our intent was to compare FPR according to test sequence when attributed with the same (if imperfect) definitions.

The most important limitation of our study is the retrospective design without randomised allocation of test sequence. While consecutive cases were extracted from a comprehensive database, the reasons for which a given patient may have undergone one test over the other first may have an impact on the prevalence of malignancy in lymph nodes and other test performance characteristics. While patients appeared similar in terms of demographics and disease stage, some slight differences in lymph node size were noted, and other unknown factors may exist even if a regression analysis was used to adjust for 
potential confounders. For example, lymph node targetting during EBUS would be mostly based on size if PETCT was not available, whereas nodal PET activity distribution may alter which nodes are sampled if those results are already available. Interestingly, both groups had similar numbers of lymph node stations sampled during the procedure, as this may relate to the nature of the procedure (diagnostic only versus staging) rather than the actual PETCT results.

In addition, since all the cases were performed with $21 \mathrm{G}$ needles, our findings should not be extrapolated to larger $19 \mathrm{G}$ devices more recently available, which could theoretically cause more trauma and inflammation $[10,11]$. Our data does not allow us to determine if there is a minimal timeline required as an interval between tests. Since our sample was limited with $<10 \%$ of lymph nodes sampled within 3 days of the PETCT, we cannot exclude that an effect may be present if EBUS precedes the PETCT examination by only a few days. Not all true positive cases were confirmed on cytopathology so that growing but benign inflammatory lymph nodes or skip metastasis could have affected our FPRs. Our study was also conducted at a single centre, and all clinicians involved in EBUS-TBNA had undergone at least one extra year of advanced training in advanced bronchoscopic techniques, including EBUS-TBNA. Thus, the homogeneity of technique and expertise at our centre may not be reflective of all centres performing EBUS-TBNA.

In summary, these results provide some reassurance to clinicians that performing EBUS prior to PETCT testing for patients undergoing investigation for possible chest malignancy should not affect results of the latter scan. This is probably less an issue in scenarios with high pretest probability of mediastinal disease where EBUS and PETCT are both likely to be positive. Importantly, the corollary is also noted that positive findings on PETCT should not be simply attributed to recent EBUS-TBNA testing and may warrant added scrutiny in light of these results. In scenarios with low pretest probability of disease (e.g. staging procedures), we still would advocate performing PETCT prior to EBUS as this may preclude need for EBUS if extrathoracic disease is identified and may impact the thoroughness of the EBUS guided by PETCT results.

The added flexibility to schedule these tests may help reduce strain on the health system and prevent delays in evaluation. These data also provide us with further evidence of the ongoing need for confirmatory testing in the case of positive PETCT results. Given an FPR of $14.6 \%$ in either groups, the possibility of inappropriate upstaging warrant confirmatory testing if important for treatment decisions.

Our results suggest that performing EBUS-TBNA within 30 days prior to PETCT does not increase the false-positive rate of PETCT for lymph node evaluation. Selection of which test to perform first should be based on other considerations.

Acknowledgements: Statistical consultation was obtained from Sarah Rose, Rho-Sigma Scientific Consultants Ltd (Bury St Edmunds, UK).

Conflict of interest: J.M. Ramsahai has nothing to disclose. C. Molnar has nothing to disclose. L. Lou has nothing to disclose. W. Ying has nothing to disclose. P. MacEachern has nothing to disclose. C.A. Hergott has nothing to disclose. E. Dumoulin has nothing to disclose. N. Strilchuk has nothing to disclose. M. Fortin has nothing to disclose.

A. Tremblay reports consulting fees from Olympus America outside the submitted work.

\section{References}

1 Jemal A, Siegel R, Xu J, et al. Cancer statistics, 2010. CA Cancer J Clin 2010; 60: 277-300.

2 Silvestri GA, Gonzalez AV, Jantz MA, et al. Methods for staging non-small cell lung cancer: diagnosis and management of lung cancer, 3rd ed: American College of Chest Physicians evidence-based clinical practice guidelines. Chest 2013; 143(5 Suppl): e211S-e250S.

3 De Wever W, Ceyssens S, Mortelmans L, et al. Additional value of PET-CT in the staging of lung cancer: comparison with CT alone, PET alone and visual correlation of PET and CT. Eur Radiol 2007; 17: 23-32.

4 Dincer HE. Linear EBUS in staging non-small cell lung cancer and diagnosing benign diseases. J Bronchology Interv Pulmonol 2013; 20: 66-76.

5 Bhargava P, Zhuang H, Kumar R, et al. Iatrogenic artifacts on whole-body F-18 FDG PET imaging. Clin Nucl Med 2004; 29: 429-439.

6 Carter KR, Kotlyarov E. Common causes of false positive F18 FDG PET/CT scans in oncology. Braz Arch Biol Technol 2007; 50: 29-35.

7 Yeung CS, Musaddaq B, Hare S, et al. 18F-FDG-PET/CT study after lung biopsy in suspected lung cancer patients: time is of the essence. Nucl Med Commun 2017; 38: 99-100.

8 Sivapalan P, Naur TMH, Colella S, et al. Impact of EBUS-TBNA on PET-CT imaging of mediastinal nodes. J Bronchology Interv Pulmonol 2017; 24: 188-192.

9 Jasra S, Roche CL, Dhillon S, et al. Quantitative false positive positron emission tomography (PET) scan following endobronchial ultrasound guided transbronchial aspiration (EBUS-TBNA): a retrospective study. Am J Respir Crit Care Med 2016; 193: A4265.

10 Tremblay A, McFadden S, Bonifazi M, et al. Endobronchial ultrasound-guided transbronchial needle aspiration with a 19-G needle device. J Bronchol Interv Pulmonol 2018; 25: 218-223.

11 Tyan C, Patel P, Czarnecka K, et al. Flexible 19-gauge endobronchial ultrasound-guided transbronchial needle aspiration needle: first experience. Respiration 2017; 94: 52-57. 